\title{
El test de la golosina en contexto: ¿cómo influye el entorno social en la toma de decisiones y el autocontrol?
}

\section{The marshmallow test in context: How does the social environment influence decision-making and self-control?}

\section{$O$ teste do marshmallow em contexto: como o ambiente social influencia a tomada de decisões e o autocontrole?}

\author{
Pablo Chaverri Chaves ${ }^{1}$, ORCID 0000-0002-2639-4242 \\ Rolando Barrantes Pereira ${ }^{2}$, ORCID 0000-0002-0969-6855 \\ L. Diego Conejo ${ }^{3}$, ORCID 0000-0002-6415-0337 \\ ${ }^{123}$ Instituto de Estudios interdisciplinarios de la Niñez y la Adolescencia (Ineina), \\ Universidad Nacional (UNA), Costa Rica
}

Resumen: Este artículo propone una discusión sobre los factores psicosociales que influyen en la manera en que los seres humanos toman decisiones relacionadas con el autocontrol, enfatizando en la forma en que lo hacen los niños preescolares en situaciones de postergación de recompensas. Para ello, toma como punto de partida el test de la golosina puesto en contexto social, para analizar la forma en la cual aspectos tales como la racionalidad, las emociones, la condición socioeconómica y particularmente la confianza en los demás influyen en cómo se toman decisiones y cómo se ejerce o no el autocontrol en relación con el postergar recompensas inmediatas para obtener, posteriormente, otras mayores.

Palabras clave: toma de decisiones; test de la golosina; autocontrol; postergación de recompensas; contexto social.

Abstract: This article proposes a discussion of psychosocial factors that influence how humans make decisions related to self-control, emphasizing how preschoolers make those decisions in delayed reward situations. To this end, it takes as a starting point the marshmallow test placed in social context, to analyze how aspects such as rationality, emotions, socioeconomic status, culture and particularly trust in others influence how decisions are made and how self-control is exercised in relation to the delay of immediate rewards to obtain, subsequently, more beneficial results.

Keywords: decision making; marshmallow test; self-control; delayed rewards; social context. 
Resumo: Este artigo propõe uma discussão sobre os fatores psicossociais que influenciam a maneira em que os seres humanos tomam decisões relacionadas ao autocontrole, enfatizando o que fazem as crianças pré-escolares em situações de adiamento de recompensa. Para isso, tomamos como ponto de partida o teste do marshmallow aplicado ao contexto social, para analisar a forma pela qual aspectos como a racionalidade, as emoções, a condição socioeconômica, e particularmente a confiança nos outros influenciam em como as decisões são tomadas e como se exerce ou não o autocontrole em relação ao adiamento de recompensas imediatas para obter, posteriormente, outras maiores.

Palavras-chave: tomada de decisão; teste do marshmallow; autocontrole; adiamento de recompensas; contexto social.

Recibido: 04/03/2021

Aceptado: 17/08/2021

Cómo citar:

Chaverri Chaves, P., Barrantes Pereira, R., \& Conejo, L. D. (2021). El test de la golosina en contexto: ¿cómo influye el entorno social en la toma de decisiones y el autocontrol? Ciencias Psicológicas, 15(2), e-2486. doi: https://doi.org/10.22235/cp.v15i2.2486

Correspondencia: Pablo Chaverri Chaves, Instituto de Estudios interdisciplinarios de la Niñez y la Adolescencia (Ineina), Universidad Nacional (UNA), Costa Rica. E-mail: pablo.chaverri.chaves@una.cr

Este artículo tiene el objetivo de analizar cómo influye el entorno social en la toma de decisiones de postergación de recompensas, poniendo la prueba de la golosina en contexto, para lo cual revisa algunos aspectos tanto conceptuales como empíricos sobre los procesos de toma de decisión relacionados con el autocontrol. Más específicamente, analiza la forma en la que la racionalidad, las emociones, la condición socioeconómica y particularmente la confianza en los demás, influyen en cómo se toman decisiones y cómo se ejerce o no el autocontrol en relación con el postergar recompensas inmediatas para obtener, posteriormente, otras mayores.

\section{El test de la golosina}

El experimento de la prueba de la golosina consiste en que a un niño o niña en edad preescolar se le ofrece un dulce (puede ser un malvavisco, una galleta, u otro similar que resulte tentador), pero se le dice que si quiere una segunda golosina debe esperar a que el experimentador regrese sin comerse la primera. Más específicamente, esta técnica consiste en que se presenta una golosina a un niño o una niña y se le dice que la misma le pertenece, pero que, si espera sin comérsela a que la persona evaluadora regrese, entonces se le entregará una segunda recompensa. Luego de esta instrucción, se le pregunta al participante si desea esperar, ante lo cual la amplia mayoría de niños y niñas en todo el mundo a los que se les ha hecho este experimento, deciden hacerlo (Mischel, 2014). 
El test de la golosina, en apariencia un simple experimento para valorar el autocontrol personal, aplicado generalmente hacia el final de la primera infancia, ha mostrado tener un valor predictivo estadísticamente significativo sobre resultados positivos a nivel educativo (más años de estudio), laboral (mejor colocación), económico (mejores ingresos), familiar (mayor estabilidad) y social (relaciones más armoniosas y menos conflictivas) en la vida adulta (Mischel, 2014; Shoda, Mischel \& Peake, 1990). En réplicas más recientes de la prueba de la golosina se ha encontrado que si bien estas predicciones muestran significancia estadística, su efecto es moderado y altamente sensible a la inclusión de controles, tales como el contexto interpersonal, cultural y socioeconómico (Lamm et al., 2018; Michaelson \& Munakata, 2016; Watts, Duncan \& Quan, 2018).

$\mathrm{Al}$ analizar a las personas décadas después de que habían realizado el experimento, la evidencia sugiere que quienes habían logrado esperar todo el tiempo dispuesto (aproximadamente 15 minutos -que pueden variar de un diseño metodológico a otro-) cuando tuvieron cerca de cinco años, tendían a presentar mejores resultados en sus vidas adultas en diversas áreas, lo cual parece apuntar a la importancia predictiva de la capacidad de autocontrol en el desarrollo, y más concretamente, de la capacidad de postergar recompensas para obtener otras mejores más tarde. Esto puede estar relacionado con la capacidad de planificar el comportamiento a largo plazo, postergando recompensas inmediatas, para poder obtener otras mayores posteriormente (Mischel, 2014).

Sin embargo, recientes réplicas de este trabajo, han venido a cuestionar su valor predictivo y su validez como técnica de medición, dadas las relativamente bajas correlaciones obtenidas entre el desempeño en la tarea de la golosina y los resultados cognitivos y sociales logrados posteriormente, así como las altas correlaciones entre el desempeño favorable de niños y niñas en esta tarea y su entorno social (alto estatus socioeconómico, vivir con ambos padres, ausencia de conflicto social severo; Watts et al., 2018).

\section{Contexto social y toma de decisiones de autocontrol}

La investigación reciente (Chaverri, Conejo, León \& Arrieta, 2020; Kidd, Palmeri \& Aslin, 2013) ha logrado determinar que el contexto social en el que se desenvuelven las personas tiene una profunda influencia sobre cómo estas toman decisiones, ya no solamente en términos generales, sino especificando instancias y dinámicas de esta interacción. En este sentido, se ha logrado demostrar que experimentar situaciones de escasez, inestabilidad ambiental y la percepción de vivir en condiciones de estatus socioeconómico bajo, tienen implicaciones directas en las habilidades cognitivas y regulatorias en contextos de atención de necesidades inmediatas, en la habilidad para resolver problemas que impliquen algún grado de razonamiento, así como en el manejo del estrés, el desempeño académico y los hábitos alimenticios (Shah, Mullainathan \& Shafir, 2012). En términos generales, se puede decir que un entorno social deteriorado relacional y materialmente puede llevar a las personas a tomar decisiones más impulsivas y a ser menos capaces de considerar una visión a largo plazo. 


\section{La confianza en las demás personas}

La confianza implica tanto a las creencias como a las emociones y es un estado emocional unido a una expectativa de comportamiento (Thagard, 2019). Confiar en otra persona implica creer que es honesta y tener un sentimiento positivo hacia esa persona. Además, Thagard (2019) propone que la confianza tiene correlatos cerebrales que une representaciones del sí mismo, del otro, de la situación y de la emoción experimentada con respecto al otro, en un patrón especial de disparo neuronal. En este sentido, confiar en las personas puede implicar estimaciones de probabilidades de cómo se comportarán, pero generalmente se confía en los demás sin predicciones precisas sobre sus comportamientos. Desde este planteamiento, el componente emocional de la confianza implica patrones neuronales que combinan representaciones de la situación de la que trata la emoción, evaluaciones de la relevancia de la situación a los objetivos del actor, percepciones de cambios fisiológicos, e incluso representaciones de sí mismo.

De forma similar, la desconfianza es un proceso emocional que va mucho más allá de la estimación de bajas probabilidades de que las personas hagan lo que se espera de ellas. También requiere representación de sí mismo, de la persona no confiable y de la situación dada, pero difiere de la confianza en que implica emociones negativas similares a la aversión y el miedo. Estas reacciones emocionales surgen de la combinación de evaluaciones cognitivas sobre objetivos insatisfechos y reacciones fisiológicas desagradables a la persona no confiable. Desconfiar de alguien no es sólo una predicción de expectativa insatisfecha, sino también una emoción sobre la persona poco confiable. En este sentido, se puede decir que la confianza es un proceso que implica la cognición y la emoción (Thagard, 2019).

En estudios recientes sobre la relación entre la confianza y el autocontrol con la prueba de la golosina (Chaverri et al., 2020; Kidd et al., 2013), se ha interpretado que la capacidad de postergar la recompensa para obtener una mayor no depende solo de considerar la cantidad de golosinas que se pueden recibir, sino también de una estimación de la probabilidad de obtenerla basada en la confianza que inspira la contraparte. De esta manera, cuando antes de hacerse la prueba de la golosina se ha roto una promesa al niño, este puede generar tanto la creencia de que el experimentador no es confiable, como un sentimiento negativo a esta persona, que se traduciría en una capacidad de espera reducida frente a la golosina.

Es decir, el efecto de espera reducida durante la prueba de la golosina no dependería solamente de que el niño o la niña represente a la contraparte como alguien que no va a cumplir la promesa de traer una segunda golosina, sino que además esta representación vendría acompañada de un sentimiento negativo hacia esta persona, lo cual llevaría al participante a lograr un tiempo menor de postergación de la recompensa. Cuando se le pide a un niño o niña que espere a cambio de una recompensa, el resultado no depende solamente de la capacidad personal de poder postergar la recompensa, sino también de qué piense y siente con respecto a la persona que le hace la promesa (Michaelson \& Munakata, 2016). Este mayor autocontrol cuando se tiene confianza en la otra persona con quien se da un intercambio podría ocurrir porque se genera sintonía interpersonal, la cual influye positivamente en la autorregulación (Siegel, 2016). Es decir, que la confianza contribuye a producir regulación interpersonal, que a su vez se convierte en una mayor autorregulación. 


\section{Las emociones}

Las emociones se pueden entender como procesos mentales y físicos que incluyen aspectos como respuestas corporales, expresiones faciales y valoración subjetiva (Smith \& Kosslin, 2012). Las emociones no pueden separarse del pensamiento y la razón cuando se analiza el comportamiento de las personas en diversas situaciones, ya que las emociones pueden ser vistas como gatilladores o inhibidores de las decisiones en la vida de las personas y a su vez las decisiones pueden verse como un reflejo de la forma en que las emociones guían los intentos individuales por anular los sentimientos negativos y por incrementar los positivos (Smith \& Kosslyn, 2012).

Esta visión de las emociones como guía invierte los papeles que tradicionalmente atribuían este rol a la racionalidad, puesto que ponen a la emoción como el ponderador de la información que la cognición permite captar, dándole una valencia negativa, positiva o neutra (Damasio, 2018). Como lo han señalado varios autores que han estudiado el papel de las emociones en la toma de decisiones (Damasio, 1994; Eagleman, 2017; Todd \& Gigerenzer, 2012), una persona con daño en regiones cerebrales dedicadas al procesamiento emocional encuentra muchas dificultades para tomar decisiones pues, aunque no tiene problemas para comprender la información disponible, no logra evaluarla y obtener así el disparo emocional que parece activar la decisión y la acción consecuente.

Contrario a lo que solía pensarse en el pasado, las emociones no serían un estorbo, ni un enemigo del razonamiento, sino que más bien parecen cumplir un papel complementario que serviría para valorar la información de la que se dispone que, al ser altamente sensible a las condiciones del entorno, podrían servir de ayuda para actuar de forma adaptativa en ese contexto.

En el caso del test de la golosina, las emociones pueden verse influidas por el contexto de confianza o desconfianza en las demás personas, influyendo en el comportamiento y en la decisión de inhibir o desencadenar la conducta de tocar o consumir la golosina. Futuros estudios deberán determinar si que cuando los niños o las niñas han recibido muestras de poca confiabilidad por parte del experimentador, esto podría generar emociones negativas relacionadas con el enojo, la frustración y el desagrado, que podrían debilitar la capacidad de postergar la recompensa, frente a una necesidad acrecentada de buscar satisfacción al consumir la golosina.

\section{La racionalidad}

Los procesos humanos de toma de decisiones en raras ocasiones se basan en el modelo racional de toma de decisiones en sentido estricto, donde las personas son consideradas como seres completamente objetivos y maximizadores de ganancias, con conocimiento pleno de las opciones que tienen a su disposición para elegir e inmunes a la influencia de eventos o emociones externas (Robbins \& Aydede, 2009). En el complejo mundo en el que nos desarrollamos, la imposibilidad de procesar grandes cantidades de información, las limitaciones de tiempo, energía y recursos cognitivos, la dificultad para clasificar nuestras preferencias y la falta de certeza en el resultado final de nuestras decisiones, hacen que los procesos de toma de decisiones se vean rodeados de elementos mucho más complejos que los meramente sugeridos por la teoría racional de toma de decisiones, entre los que destacan la condición socioeconómica, la cultura e incluso los 
modelos de crianza que eventualmente pueden influir en cómo tomamos decisiones (Lamm et al., 2018).

En este sentido, la teoría ecológica de la toma de decisiones posibilita pensar la forma en que la interacción entre las personas y sus entornos da forma a la manera en que estas toman decisiones. Así pues, la racionalidad ya no sería un simple cálculo de utilidad y probabilidades de obtener ganancias, sino que se trata de una interacción compleja entre el individuo y su entorno que considera diversos aspectos de la situación particular y del contexto más amplio. Entonces, comportarse en línea con los principios de la racionalidad clásica y comportarse de forma adaptativa, no serían necesariamente el mismo proceso. Una mejor comprensión de los sistemas cognitivos humanos necesita una visión más ecológica de la racionalidad, donde las limitaciones cognitivas y los contextos específicos sean vistos como aspectos significativos de una cognición adaptativa, en lugar de una perspectiva del ser humano como un robot calculador adherido a los principios de la racionalidad clásica, incapaz de considerar diversos aspectos particulares de cada contexto físico y social (Robbins $\&$ Aydede, 2009). Sin duda, esta línea de investigación debe ser abordada por futuros estudios en las ciencias del desarrollo y la educación, si pretendemos lograr modelos explicativos del comportamiento de niñas y niños que nos permitan derivar intervenciones psicosociales con validez ecológica y pertinencia social.

\section{La cognición situada}

Los resultados en el test de la golosina contextualizado aquí referidos (Chaverri et al., 2020; Kidd et al, 2013), muestran que las y los niños en condiciones más confiables y estables logran mejores resultados en la postergación de la recompensa. Esta interacción entre el niño y su entorno podrían encontrar mayor sentido desde la perspectiva de la teoría ecológica de la toma de decisiones, la cual asume que la cognición no es un proceso mental aislado del entorno en el que se encuentra la persona, sino que más bien se trata de un proceso en el que la actividad mental aprovecha la estructura del ambiente físico y social (Robbins \& Aydede, 2009). A esta forma de entender la cognición como algo integrado al entorno se le conoce como cognición situada, y la racionalidad ecológica puede ser entendida como una forma de cognición situada (Brighton \& Todd, 2009).

La racionalidad ecológica depende de agentes que usan varias estrategias de decisión de forma sensible al ambiente en el que están inmersos. La cognición situada mira el comportamiento humano como algo inmerso social y materialmente, que emerge dentro de los detalles concretos y específicos de entornos particulares, más que como un proceso abstracto, desconectado y de propósito general de racionalización lógica.

Volviendo al experimento de la golosina en contexto, mientras el comportamiento más impulsivo de quienes esperan menos tiempo en esta tarea puede ser visto como menos racional desde la perspectiva de la teoría racional de la toma de decisiones, este podría ser visto como ecológicamente racional cuando antes de la prueba de la golosina el experimentador ha dado muestras de no ser confiable, puesto que ha roto su promesa previa de traer materiales mejorados para dibujar. Es decir, desde una perspectiva situada, un comportamiento que podría ser catalogado como irracional, pasaría a verse como ecológicamente racional si contribuye a responder a las circunstancias en las que se encuentra el decisor. Otro aspecto relevante para este planteamiento es que el menor tiempo de espera también se relaciona con la condición socioeconómica de las personas, lo cual sugiere que 
no solo el contexto inmediato de la decisión, sino también el contexto de procedencia influye en la experiencia de la persona, su percepción, su sentir y su actuar frente a la tarea de la golosina puesta en contexto.

\section{La interacción entre la racionalidad y la emoción}

Tradicionalmente, la literatura sobre toma de decisiones equiparó la racionalidad con el maximizar el valor de una potencial ganancia (Smith \& Kosslyn, 2012; Sternberg, Espinosa Rodríguez, Ortíz Salinas \& Reyes Ponce, 2011). Este modelo de utilidad esperada partía de tres supuestos básicos sobre la persona que toma la decisión: 1) está totalmente informada acerca de todas las posibles opciones de elección, 2) es muy sensible a distinciones sutiles entre las opciones existentes, 3) es totalmente racional respecto a la elección entre opciones (Slovic, 1990).

Consistentemente, obtener buenos resultados en el test de la golosina fue explicado desde la teoría racional de la toma de decisiones (Mischel \& Staub, 1965), partiendo de que lo que este experimento hace es comparar una elección entre una gratificación inmediata menor y una gratificación posterior mayor, lo cual se relaciona con la idea de las personas como agentes magnificadores de utilidades, propia de la teoría racional de la toma de decisiones, por lo que una mayor espera se podría explicar dentro en esta teoría en términos de una mayor racionalidad (Kidd et al., 2013).

Pero: ¿es solo la racionalidad lo que explica las diferencias? De acuerdo con la teoría racional de la toma de decisiones, los seres humanos son agentes que toman decisiones de manera utilitaria, buscando incrementar beneficios y reducir pérdidas (Smith \& Kosslyn, 2012). Sin embargo, la investigación más reciente en economía del comportamiento, psicología y neurociencias cognitivas ha venido demostrando que las personas son, con mucha mayor frecuencia de lo que se suele suponer, tomadores poco racionales de decisiones (Ariely, 2010; Kahneman, 2011), siempre que la racionalidad sea definida en los términos de los supuestos del modelo de utilidad esperada.

Una línea de investigación que pone esta cuestión en perspectiva es el "juego del ultimátum" (Jensen, Call \& Tomasello, 2007). En este juego, dos personas, sentadas una frente a la otra, deben decidir cómo distribuir unos recursos entregados a ellas. Se entregan, por ejemplo, diez billetes de la misma denominación a uno de los jugadores (el oferente), quien debe decidir cómo distribuirlos con su contraparte, siendo que, si el receptor rechaza el trato, ambos jugadores se quedan sin nada. Por otro lado, si el receptor acepta el trato, entonces ambos jugadores se quedan con lo que propuso el jugador oferente (Güth, Schmittberger \& Schwarze, 1982).

Siguiendo el modelo de la utilidad esperada sobre la racionalidad en la toma de decisiones, las personas jugadoras deberían rechazar toda oferta igual a cero (porque implica no obtener ganancia alguna) y aceptar toda oferta igual o superior a uno (porque implica mejorar la situación original que es cero). Sin embargo, las personas no actúan así, ya que, en una distribución con un total de 10 unidades, tienden a rechazar aquellas ofertas en las que les asignan dos o menos unidades y aceptar las que les hacen ganar tres unidades o más (Jensen et al., 2007). Además, si la oferta es dada por una persona, el receptor tiende a un mayor rechazo de ofertas bajas (8:2 o 9:1) que si el oferente es una computadora, en cuyo 
caso se da una mayor aceptación de ofertas bajas, lo cual sugiere la influencia de un juicio sobre la capacidad moral de la contraparte (Fehr, 2009).

Este comportamiento de toma de decisiones no encaja con lo esperado por la teoría racional, de que se acepten todas las ofertas que impliquen una ganancia. ¿Por qué ocurre esto? Una posibilidad que cuenta con evidencia de respaldo es que cuando las personas se encuentran en contextos de interdependencia entre ellas, en lugar de clasificar las ofertas de forma meramente racional y utilitaria, parece más bien ocurrir una ponderación más emocional y presumiblemente moral (Sanfey, Rilling, Aronson, Nystrom \& Cohen, 2003). Lo anterior es respaldado por el hallazgo ya mencionado de que cuando el intercambio se hace con respecto a una computadora, tiende a haber un menor rechazo de ofertas que cuando se juega con otra persona, lo cual sugiere un juicio moral sobre las intenciones de la contraparte. Por otro lado, cuando se reciben ofertas bajas (que serían consideradas injustas en un contexto interpersonal) se presenta una mayor activación de áreas cerebrales asociadas con el procesamiento emocional (por ejemplo la ínsula anterior derecha) en comparación con el procesamiento cerebral de información en ofertas igualitarias. Esto sugiere que existe un rol aumentado de la emoción cuando se recibe una oferta desigual. Además, las personas con una mayor activación de la ínsula anterior derecha frente a ofertas bajas rechazan en mayor proporción tales ofertas (Sanfey et al., 2003), lo que sugiere una interacción entre este procesamiento emocional y el rechazo.

En el estudio realizado por Sanfey et al. (2003), se escaneó el cerebro a participantes del juego del ultimátum con imágenes de resonancia magnética funcional mientras respondían a propuestas catalogadas como justas (que tienden a la paridad 50/50) e injustas (que se acercan más a disparidad total 0/100). Las ofertas injustas provocaron actividad en áreas cerebrales relacionadas tanto con la emoción (ínsula anterior) como con la cognición (corteza prefrontal dorsolateral). Esta evidencia sugiere un papel importante de las emociones en esta toma de decisiones.

Entonces, contrario a lo que predice el modelo de la utilidad esperada, la toma de decisiones en contextos interpersonales, lejos de ser un proceso exclusivamente utilitario, consiste en un proceso mediado emocional y moralmente, idea que se ha venido fortaleciendo en los últimos años gracias a investigaciones que tratan de entender la mente y el comportamiento humano dentro de su entorno, analizando interacciones específicas, así como el papel de las emociones, que no están ahí para interrumpir, distraer o inducir a error, sino que son importantes para las funciones cognitivas y la toma de decisiones de manera contextualizada, aunque todavía falte mucho por comprender al respecto (Damasio, 2018; Todd \& Gigerenzer, 2012). Dado lo anterior, se puede argumentar que la investigación reciente sugiere que las emociones, la moralidad y el contexto social son factores importantes por considerar para comprender la forma en que las personas toman decisiones y ejercen sus habilidades de autocontrol, sin negar esto la importancia de la cognición y el razonamiento, sino más bien reconociendo la necesidad de lograr una comprensión más profunda de cómo interactúan estos elementos entre sí. 
Como plantean Todd y Gigerenzer (2012), el futuro de la investigación sobre toma de decisiones debería entender estas interacciones entre la mente y su entorno, pero esto no coincide con la postura de aquellos científicos sociales más apegados a la teoría tradicional de la elección racional. En criterio de estos autores, entendemos muy poco la relación entre las decisiones y las emociones, y sobre cómo desarrollamos un buen repertorio para tomar decisiones, por lo que es necesario fortalecer la investigación en esta dirección.

\section{Conclusión}

Según lo sugiere la investigación aquí revisada, el autocontrol no puede considerarse una capacidad independiente del entorno, pues está influido por las circunstancias que envuelven a las personas, siendo la confianza interpersonal un aspecto relevante de esta interacción persona-entorno.

Cuando un niño o niña decide no esperar por el segundo dulce después de recibir un engaño de su contraparte (como ocurre en el experimento aquí revisado cuando se incumple una promesa), en lugar de juzgar su acto como irracional, más bien se debería considerar el contexto emocional que produce la desconfianza inducida, para comprender la forma en que la acción del niño o niña se adapta a la estructura de su entorno, como lo proponen la teoría de la cognición situada y la teoría de la racionalidad ecológica. Cuando una persona no se siente emocionalmente cómoda en una situación dada, posiblemente sea apropiado tratar de ver qué es lo que esa emoción negativa significa en su contexto, que simplemente tratar de inhibirla por considerarla "un obstáculo" al razonamiento.

Que una persona en un contexto más inseguro, inestable y carenciado actúe de forma más impulsiva y muestre más dificultad para postergar una recompensa, podría ser considerado una respuesta adaptativa a tales características de su contexto, pues se podría decir que su experiencia le ha enseñado que no se puede confiar a priori en las promesas de los demás y de su entorno, puesto que vive en un ambiente donde estas se suelen romper con facilidad y las expectativas quedan incumplidas. ¿Es esta persona menos racional? Si se toma en consideración la interacción entre la persona y su entorno, como lo propone la teoría de la racionalidad ecológica y la cognición situada, se podrá ver que no esperar por la segunda golosina puede ser considerado un comportamiento adaptativo en algunas circunstancias.

Cuando se toma en cuenta el contexto de una persona, se puede comprender que alguien que vive en una situación más segura, estable y satisfactoria, podría conseguir más fácilmente inhibir sus impulsos de satisfacción inmediata y esperar, ya que puede tener más esperanza de que lo prometido se cumplirá, pues está más habituada a que así sea. Ofrecer entonces ambientes comunales y educativos que fortalezcan el sentido de estabilidad y confianza básica de las niñas y los niños, resulta fundamental para promover su capacidad de autocontrol, toma de decisiones y conductas adaptativas. 


\section{Referencias}

Ariely, D. (2010). Predictably irrational: The hidden forces that shape our decisions (Revisado y ampliado). Harper Perennial.

Brighton, H. \& Todd, P. (2009). Situationg rationality: ecologically rational decision making with simple heuristics. En Robbins, P., \& Aydede, M. (Eds.), The Cambridge Handbook of Situated Cognition (pp. 322-346). Cambridge: Cambridge University Press.

Chaverri, P., Conejo, D., León, S., \& Arrieta, A. (2020). Postergación de la gratificación en preescolares costarricenses: efecto de la confianza en la persona experimentadora y del estrato socioeconómico. Artículo en revisión.

Damasio, A. R. (1994). Descartes' error: Emotion, reason, and the human brain. Putnam.

Damasio, A. (2018). El extraño orden de las cosas: La vida, los sentimientos y la creación de las culturas. Ediciones Destino.

Eagleman, D. (2017). The brain: The story of you. Vintage Penguin Random House.

Fehr, E. (2009). Social Preferences and the Brain. En P. W. Glimcher, C. F. Camerer, E. Fehr, \& R. A. Poldrack (Eds.), Neuroeconomics (pp. 215-232). Academic Press. doi: https://doi.org/10.1016/B978-0-12-374176-9.00015-4

Güth, W., Schmittberger, R., \& Schwarze, B. (1982). An experimental analysis of ultimatum bargaining. Journal of Economic Behavior \& Organization, 3(4), 367-388. doi: https://doi.org/10.1016/0167-2681(82)90011-7

Jensen, K., Call, J., \& Tomasello, M. (2007). Chimpanzees Are Rational Maximizers in an Ultimatum Game. Science, 318(5847), 107-109. doi: https://doi.org/10.1126/science. 1145850

Kahneman, D. (2011). Thinking, fast and slow (1 ${ }^{\circ}$ ed.). Farrar, Straus and Giroux.

Kidd, C., Palmeri, H., \& Aslin, R. N. (2013). Rational snacking: Young children's decisionmaking on the marshmallow task is moderated by beliefs about environmental reliability. Cognition, 126(1), 109-114. doi: https://doi.org/10.1016/j.cognition.2012.08.004

Lamm, B., Keller, H., Teiser, J., Yovsi, R., Suhrke, J., Vohringer, I., Knopf, M., Lohaus, A., Gudi, H., Freitag, C., Fassbender, I., Teubert, M. \& Schwarzer, G. (2018). Waiting for the Second Treat: Developing Culture-Specific Modes of Self-Regulation. Child Development, 89(3), e261-e277. doi: https://doi.org/10.1111/cdev.12847

Michaelson, L. E., \& Munakata, Y. (2016). Trust matters: Seeing how an adult treats another person influences preschoolers' willingness to delay gratification. Developmental Science, 19(6), 1011-1019. doi: https://doi.org/10.1111/desc.12388

Mischel, W. (2014). The marshmallow test: Mastering self-control. Little, Brown and Company.

Mischel, W., \& Staub, E. (1965). Effects of expectancy on working and waiting for larger rewards. Journal of Personality and Social Psychology, 2, 625-633. doi: https://doi.org/10.1037/h0022677

Robbins, P., \& Aydede, M. (Eds.). (2009). The Cambridge handbook of situated cognition. Cambridge University Press. 
Sanfey, A. G., Rilling, J. K., Aronson, J. A., Nystrom, L. E., \& Cohen, J. D. (2003). The Neural Basis of Economic Decision-Making in the Ultimatum Game. Science, 300(5626), 1755-1758. doi: https://doi.org/10.1126/science.1082976

Siegel, D. J. (2016). Guía de neurobiología interpersonal: Un manual integrativo de la mente. Eleftheria.

Shah, A. K., Mullainathan, S., \& Shafir, E. (2012). Some consequences of having too little. Science, 338(6107), 682-685. doi: https://doi.org/10.1126/science.1222426

Shoda, Y., Mischel, W., \& Peake, P. K. (1990). Predicting adolescent cognitive and social competence from preschool delay of gratification: Identifying diagnostic conditions. Developmental Psychology, 26, 978-986. doi: https://doi.org/10.1037/00121649.26.6.978

Slovic, P. (1990). Choice. En: D. Osherson, E. Smith (Eds.). An invitation to cognitive science: Vol. 3. Thinking (pp. 89-116). MIT Press.

Smith, E. E. \& Kosslyn, S. M. (2012). Procesos cognitivos: Modelos y bases neuronales. Pearson Prentice Hall.

Sternberg, R. J., Espinosa Rodríguez, J., Ortíz Salinas, M. E., \& Reyes Ponce, L. (2011). Psicología Cognoscitiva. Cengage Learning.

Thagard, P. (2019). Mind-society: From brains to social sciences and professions. Oxford University Press. doi: https://doi.org/10.1093/oso/9780190678722.001.0001

Todd, P. M., \& Gigerenzer, G. (Eds.). (2012). Ecological rationality: Intelligence in the world. Oxford University Press. https://doi.org/10.1093/acprof:oso/9780195315448.001.0001

Watts, T. W., Duncan, G. J., \& Quan, H. (2018). Revisiting the Marshmallow Test: A Conceptual Replication Investigating Links Between Early Delay of Gratification and Later Outcomes. Psychological Science, 29(7), 1159-1177. doi: https://doi.org/10.1177/0956797618761661

Contribución de los autores: a) Concepción y diseño del trabajo; b) Adquisición de datos; c) Análisis e interpretación de datos; d) Redacción del manuscrito; e) revisión crítica del manuscrito.

P. C. C. ha contribuido en a, b, c, d, e; R. B. P. en a, b, c, d, e; L. D. C. en a, b, c, d, e.

Editora científica responsable: Dra. Cecilia Cracco. 